\title{
A Study on the Correlation between Levels of Serum Vitamin D and Pre-Diabetic Individuals
}

\author{
Manglik CG ${ }^{1}$, Batra $\mathrm{CM}^{2}$ and Harplani $\mathrm{BD}^{3}$ \\ ${ }^{1}$ Foods and Nutrition, Shri Venkateshwara University, India \\ ${ }^{2}$ Endocrinologist, Indraprastha Apollo Hospital, India \\ ${ }^{3}$ Foods and Nutrition, Shri Venkateshwara University, India
}

\section{Mini review}

Volume 2 Issue 3

Received Date: September 06, 2018

Published Date: September 27, 2018

*Corresponding author: Chhavi Goel Manglik, Foods and Nutrition, Shri Venkateshwara University, India, Tel: +918826386125; Email: chhavi.aspect@gmail.com

\section{Abstract}

Diabetes and vitamin D are pandemic diseases. To control the incidences of illness a measure is taken to control it before the diagnoses of it. Vitamin D deficiency leads to insulin resistance. Its supplementation in pre-diabetes individuals has shown the association with considerably drop in progression to diabetes in between the age 15 yrs to 55 years. The participants were screened thoroughly and then taken their written consent before enrolling them into the side. No individual was forced for the enrollment into the study. No Type I Diabetes, Type II Diabetes and GDM cases were included. The study was done only on pre-diabetics who were not on medicines. Moreover, only vitamin D supplementation was given orally or injectable as per the patient's choice. The supplementation has shown the reversal of pre-diabetics to normal. The study showed the significant increase in vitamin D and serum calcium levels was observed post intervention in both males and females. There was a significant reduction in fasting blood sugar, post prandial blood sugar and HbA1C post intervention in both males and females indicating that vitamin D supplementation has a beneficial role in the management of insulin sensitivity and blood glucose levels in pre-diabetics $(p<0.001)$.

Keywords: Vitamin D; Diabetes; Blood sugar; Glucose; Insulin

\section{Introduction}

The population of India is deficient in vitamin $\mathrm{D}$ and the incidences reported for pre-diabetes has increased tremendously. Pre-diabetes is associated with the simultaneous presence of insulin resistance and $\beta$-cell dysfunction, abnormalities that start before glucose changes are detectable. Observational evidence shows associations of pre-diabetes with early forms of nephropathy, chronic kidney disease, small fiber neuropathy, diabetic retinopathy, and increased risk of macro-vascular disease. Multifactorial risk scores could optimize the estimation of diabetes risk using noninvasive parameters and blood-based metabolic traits in addition to glycemic values. For pre-diabetic individuals, lifestyle modification is the cornerstone of diabetes prevention with evidence of a $40 \%-70 \%$ relative risk reduction. 


\section{Open Access Journal of Endocrinology}

\section{Review}

In 2012, Dutta, et al. studied to predict the connection of serum vitamin D and insulin resistance connection in pre-diabetic individuals. He took 115 individual and screened them and concluded that there is some connection between the two; in India, where the CVD risk is on higher levels. He further stated the prospective study is needed to approve the verdicts [1].

In a 2012 review, researchers looked at studies examining how much vitamin D people and were followed to see if they got T2D later in life. The study concluded people with the highest vitamin D blood levels had a $19 \%$ decreased chance of developing T2D compared to those with the lowest levels [2].

Low serum vitamin D levels have been associated with insulin resistance, metabolic syndrome, and type 2 diabetes, and many non-Western immigrants in the Netherlands are vitamin D deficient, obese, and at high risk of diabetes, explained Paul Lips, $\mathrm{MD}, \mathrm{PhD}$, from the VU University Medical Center in Amsterdam [3].

Kabadi et al, in his study has proved that the collaboration between vitamin $\mathrm{D}$ and body mass index (BMI) has approx. $47 \%$ of raised chances of people to be diagnosed as insulin resistant [4].

Clemente-Postigo and coworkers who announced pointedly the VDD in pre-diabetic and diabetic patients while relating it with an individual irrespective of uncontrolled glycemic status. This was neutral towards BMI [5].

\section{Methodology}

The study is aimed to see whether the good amount of vitamin $D$ improvise the living being standard by controlling the incidences of vitamin D or not. The study is done in a same institutions. Each enrolled individual is checked for the inclusion and exclusion criteria. Written consent was signed before enrolling them. All parameters are documented very aptly. The blood samples were taken on the first day of their enrolment for BS (F), (PP), HbA1c, 25(OH)D, Serum Calcium. The dose of 60K IU for vitamin D once a week for 10 weeks and $500 \mathrm{mg}$ elemental calcium twice a day for 10 weeks was recommended. And the same tests were repeated after 10 weeks. Vitamin D test was done by sandwich ELISA method. Serum Calcium was done by ARSENAZO III. HbA1c was done by HPLC method and both the blood glucose levels (fasting and post prandial) were done by using Hexokinase.

\section{Results}

There was a significant reduction in fasting blood sugar, post prandial blood sugar and HbA1c postintervention in both males and females indicating that vitamin D supplementation has a beneficial role in management of insulin sensitivity and blood glucose levels in pre-diabetics $(\mathrm{p}<0.001)$. There was no significant difference in blood parameters between males and females post intervention ( $p>0.05)$ (Table 1$)$.

\begin{tabular}{|c|c|c|c|c|c|c|}
\hline & \multicolumn{3}{|c|}{ Males (n=78) } & \multicolumn{3}{c|}{ Females (n=74) } \\
\hline & Pre-intervention & Post-intervention & P value & Pre-intervention & Post-intervention & P value \\
\hline Fasting blood sugar (mg/dl) & $110.9 \pm 8.5$ & $90.4 \pm 7.5$ & 0.001 & $114.4 \pm 9.5$ & $90.0 \pm 6.7$ & 0.001 \\
\hline $\begin{array}{c}\text { Post prandial blood sugar } \\
\text { (mg/dl) }\end{array}$ & $153.7 \pm 23.2$ & $118.7 \pm 11.0$ & 0.001 & $158.5 \pm 23.2$ & $120.8 \pm 13.1$ & 0.001 \\
\hline HbA1c (\%) & $6.1 \pm 0.2$ & $5.1 \pm 0.2$ & 0.001 & $6.2 \pm 0.2$ & $5.1 \pm 0.2$ & 0.001 \\
\hline $\begin{array}{c}\text { Serum 25-hydroxy vitamin D } \\
\text { (ng/ml) }\end{array}$ & $11.8 \pm 9.2$ & $40.9 \pm 9.0$ & 0.001 & $13.2 \pm 9.9$ & $38.7 \pm 6.4$ & 0.001 \\
\hline Serum total calcium (mg/ml) & $8.5 \pm 0.4$ & $9.1 \pm 0.3$ & 0.001 & $8.7 \pm 0.6$ & $9.2 \pm 0.4$ & 0.001 \\
\hline
\end{tabular}

Table1: Blood parameters pre and post intervention in pre-diabetics when classified according to gender. Data presented as Mean \pm SD.

There was a significantly higher decrease in fasting blood sugar in females as compared to males $(\mathrm{p}<0.05)($ Table 2). 


\section{Open Access Journal of Endocrinology}

\begin{tabular}{|c|c|c|c|}
\hline & Males (n=78) & Females (n=74) & P value \\
\hline Change in Fasting blood sugar (\%) & $18.1 \pm 8.5$ & $20.9 \pm 8.1$ & 0.047 \\
\hline Change in Post prandial blood sugar (\%) & $21.2 \pm 14.5$ & $22.7 \pm 11.7$ & 0.466 \\
\hline Change in HbA1c (\%) & $18.6 \pm 12.7$ & $16.7 \pm 3.6$ & 0.208 \\
\hline Change in Serum 25-hydroxy vitamin D (\%) & $403.1 \pm 363$ & $354.5 \pm 265.2$ & 0.367 \\
\hline Change in Serum total calcium (\%) & $7.1 \pm 6.1$ & $5.7 \pm 7.8$ & 0.202 \\
\hline
\end{tabular}

Table 2: Percentage change in blood parameters when classified according to gender.

Data presented as Mean \pm SD

As seen in Table 3, there was a significant reduction in fasting blood sugar, post prandial blood sugar and HbA1c post-intervention in all $3 \mathrm{BMI}$ categories indicating that vitamin D supplementation has a beneficial role in management of insulin sensitivity and blood glucose levels in pre-diabetics irrespective of weight categories

$(\mathrm{p}<0.001)$. A significant increase in vitamin $\mathrm{D}$ and serum calcium levels was observed post intervention in all $3 \mathrm{BMI}$ categories $(\mathrm{p}<0.001)$ (Table 3).

Data presented as Mean \pm Sd. ${ }^{*} \mathrm{p}<0.05$ for comparison between normal weight and obese pre-diabetics.

\begin{tabular}{|c|c|c|c|c|c|c|c|c|c|}
\hline & \multicolumn{3}{|c|}{ Normal weight (n=28) } & \multicolumn{2}{c|}{ Overweight (n=27) } & \multicolumn{3}{c|}{ Obese (n=97) } \\
\hline & $\begin{array}{c}\text { Pre- } \\
\text { intervention }\end{array}$ & $\begin{array}{c}\text { Post- } \\
\text { Pntervention }\end{array}$ & P value & $\begin{array}{c}\text { Pre- } \\
\text { intervention }\end{array}$ & $\begin{array}{c}\text { Post- } \\
\text { Pntervention }\end{array}$ & P value & $\begin{array}{c}\text { Pre- } \\
\text { intervention }\end{array}$ & $\begin{array}{c}\text { Post- } \\
\text { intervention }\end{array}$ & P value \\
\hline $\begin{array}{c}\text { Fasting blood sugar } \\
\text { (mg/dl) }\end{array}$ & $114.1 \pm 10.3$ & $88.9 \pm 7.3$ & 0.001 & $110.7 \pm 9.7$ & $90.1 \pm 7.4$ & 0.001 & $112.8 \pm 8.6$ & $90.6 \pm 7.0$ & 0.001 \\
\hline $\begin{array}{c}\text { Post prandial blood } \\
\text { sugar (mg/dl) }\end{array}$ & $147.2 \pm 26.3$ & $120.9 \pm 10.8$ & 0.001 & $151.2 \pm 18.3$ & $118.4 \pm 13.1$ & 0.001 & $159.9 \pm 22.8 *$ & $119.8 \pm 12.3$ & 0.001 \\
\hline HbA1c (\%) & $6.2 \pm 0.2$ & $5.2 \pm 0.2$ & 0.001 & $6.1 \pm 0.2$ & $5.0 \pm 0.2$ & 0.001 & $6.1 \pm 0.2$ & $5.1 \pm 0.2$ & 0.001 \\
\hline $\begin{array}{c}\text { Serum 25-hydroxy } \\
\text { vitamin D (ng/ml) }\end{array}$ & $12.2 \pm 8.9$ & $38.2 \pm 7.7$ & 0.001 & $13.4 \pm 10.1$ & $41.2 \pm 6.5$ & 0.001 & $12.3 \pm 9.7$ & $40.0 \pm 8.2$ & 0.001 \\
\hline $\begin{array}{c}\text { Serum total calcium } \\
\text { (mg/ml) }\end{array}$ & $8.7 \pm 0.7$ & $9.1 \pm 0.3$ & 0.001 & $8.6 \pm 0.5$ & $9.1 \pm 0.3$ & 0.001 & $8.6 \pm 0.5$ & $9.1 \pm 0.3$ & 0.001 \\
\hline
\end{tabular}

Table 3: Change in blood parameters in pre-diabetics when classified according to Nutritional Status (BMI Categories).

Table 4, significantly higher percentage decrease in post-prandial blood sugar was observed in obese prediabetics as compared to normal weight pre-diabetics indicating that vitamin D supplementations has a greater effect on controlling blood sugar levels in obese prediabetics (Table 4) $(\mathrm{p}<0.05)$.

\begin{tabular}{|c|c|c|c|}
\hline & Normal weight $(\mathrm{n}=28)$ & Over weight $(n=27)$ & Obese $(n=97)$ \\
\hline Change in Fasting blood sugar (\%) & $21.6 \pm 1.7$ & $18.1 \pm 1.6$ & $19.2 \pm 0.8$ \\
\hline Change in Post prandial blood sugar (\%) & $16.2 \pm 3.0$ & $21.2 \pm 1.6$ & $23.8 \pm 1.3^{*}$ \\
\hline Change in HbA1c (\%) & $16.9 \pm 0.6$ & $17.4 \pm 0.7$ & $17.9 \pm 1.2$ \\
\hline Change in Serum 25-hydroxy vitamin D (\%) & $402.7 \pm 66$ & $245.3 \pm 59$ & $382.2 \pm 34.1$ \\
\hline Change in Serum total calcium (\%) & $6.2 \pm 1.7$ & $5.6 \pm 1.5$ & $6.7 \pm 0.6$ \\
\hline
\end{tabular}

Table 4: Percentage change in blood parameters when classified according to nutritional status.

Data presented as Mean \pm SE. ${ }^{*} \mathrm{p}<0.05$ for comparison between normal weight and obese pre-diabetics

\section{Conclusion}

The study concluded that vitamin D stimulates pancreas to make insulin, which either prolongs the duration of onset of type II DM, or helps in reverting the impaired glucose tolerance levels or impaired fasting glucose levels into normal levels. The study showed the significant increase in vitamin $\mathrm{D}$ and serum calcium levels was observed post intervention in both males and females. There was a significant reduction in fasting blood 


\section{Open Access Journal of Endocrinology}

sugar, post prandial blood sugar and $\mathrm{HbA}_{1} \mathrm{C}$ post intervention in both males and females indicating that vitamin $\mathrm{D}$ supplementation has a beneficial role in the management of insulin sensitivity and blood glucose levels in pre-diabetics $(\mathrm{p}<0.001)$.

\section{Limitations of the Study}

1. 24 hours recall method will be used for data collection three consecutive days only.

2. Verification of the authenticity of answers regarding the amount and type of food consumed will not be possible.

3. Dietary recall was purely based on their statement.

4. Seasonal variation in the dietary intake was not taken into consideration due to limited time period of the study.

\section{Scope of the Study}

1. The scope of the study indicates:

2. The requirement and role of calcium.

3. The requirement and role of vitamin $D$.

4. The need of medicines in pre-diabetes.

5 . The requirement and role of diet in controlling prediabetes.

6. The requirement and role of phosphorus.

7. The requirement and role of magnesium.

8. The need of exercise or any other type of physical activity.

\section{Significance of the Study}

1. The significance of the study is to:

2. Find the effect of Vitamin D in decreasing insulin resistance.
3. Find the effect of Vitamin D in enhancing insulin sensitivity.

4. Find the effect of Calcium in decreasing insulin resistance.

5. Find the effect of Calcium in enhancing insulin sensitivity.

6. Role of diet.

7. Role of physical activity.

\section{References}

1. Dutta D, Maisnam I, Shrivastava A, Sinha A, Ghosh S, et al. (2013) Serum vitamin-D predicts insulin resistance in individuals with pre-diabetes. Indian J Med Res 138(6): 853-860.

2. Mitri J, Muraru MD, Pittas AG (2011) Vitamin D and type 2 diabetes: a systematic review. Eur J Clin Nutr 65(9): 1005-1015.

3. Parildar H, Cigerli O, Unal DA, Gulmez O, Demirag NG, et al. (2013) The impact of Vitamin D Replacement on Glucose Metabolism. Pak J Med Sci 29(6): 1311-1314.

4. Kabadi SM, Lee BK, Liu L (2012) Joint effects of obesity and vitamin D insufficiency on insulin resistance and type 2 diabetes: results from the NHANES 2001-2006. Diabetes Care 35(10): 20482054.

5. Clemente-Postigo M, Munoz-Garach A, Serrano M, Garrido-Sanchez L, Bernal-Lopez MR, et al. (2015) Serum 25-hydroxyvitamin $\mathrm{D}$ and adipose tissue vitamin D receptor gene expression: relationship with obesity and type 2 diabetes. J Clin Endocrinol Metab 100(4): E591-E595.

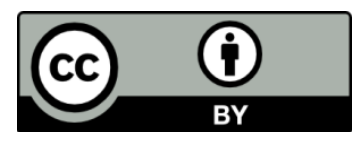

Manglik CG, et al. A Study on the Correlation between Levels of Serum Vitamin D and Pre-Diabetic Individuals. J Endocrinol 2018, 2(3): 000131. 